\title{
Lattice Boltzmann models for non-equilibrium gas flows
}

\author{
Gui-Hua Tang \\ Computational Science and Engineering Department, \\ Daresbury Laboratory, Warrington, WA4 4AD, UK \\ Yong-Hao Zhang* \\ Department of Mechanical Engineering, \\ University of Strathclyde, Glasgow, G1 1XJ, UK \\ David R. Emerson \\ Computational Science and Engineering Department, \\ Daresbury Laboratory, Warrington, WA4 4AD, UK
}

(Dated: April 17, 2008)

\begin{abstract}
Due to its computational efficiency, the kinetic-based lattice Boltzmann method has recently been used to model non-equilibrium gas dynamics. With appropriate boundary conditions, lattice Boltzmann models have been able to capture both velocity slip and temperature jump at a solid surface. To enable these models to simulate flows in the transition regime, both high-order and modified lattice Boltzmann models have been proposed. In this paper, we demonstrate the advantages of the standard lattice Bhatnagar-Gross-Krook model in predicting high-order rarefaction phenomenon. In addition, we show that current high-order lattice Boltzmann models are not yet able to capture the nonlinear constitutive relation for the stress in the Knudsen layer, despite the improved predictions of the wall slip-velocity, especially for Poiseuille flow. By considering how the wall affects the gas mean free path, we present a simplified high-order lattice Boltzmann model that can predict flow in the transition regime and is also able to capture the essential characteristics of the Knudsen layer.
\end{abstract}

PACS numbers: 05.10.-a, 47.45.-n, 47.60.+i

\footnotetext{
* Author to whom correspondence should be addressed. Email: yonghao.zhang@strath.ac.uk
} 


\section{INTRODUCTION}

Gas flows in micro/nano-fluidic devices have found a broad range of applications, from extracting biological samples to active aerodynamic flow control. Flows in these miniaturized devices are generally low speed and often exhibit non-equilibrium phenomena. As these flows are no longer in local thermodynamic quasi-equilibrium, the linear constitutive relation for stress, which is assumed in the Navier-Stokes equations, is no longer valid. Whether a gas flow is in local equilibrium or not can be determined by the Knudsen number, which is defined as

$$
K n=\frac{\lambda}{L} \approx \frac{\lambda}{\Phi}\left|\frac{d \Phi}{d L}\right|,
$$

where $\lambda$ is the mean free path of the gas molecules, $L$ is a characteristic length scale, and $\Phi$ is a quantity of interest, such as the gas density, pressure or temperature. When the mean free path of the gas molecules approaches the length scale of the system, effects occurring at the micro-scale and the macro-scale become increasingly coupled. This lack of scale separation and local disequilibrium leads to the failure of the Navier-Stokes equations.

The Navier-Stokes equations with no-velocity-slip and no-temperature-jump wall conditions are only appropriate when $K n<0.001$. Gas flows in micro/nano-fluidic devices are often in the slip $(0.001<K n<0.1)$ or the transition regime $(0.1<K n<10)$. In these flow regimes, the gas cannot properly be described as a continuous quasi-equilibrium fluid, nor as a free molecular flow. In practice, most devices will operate with a range of Knudsen numbers in different parts of the device; this makes it even more difficult to develop a generalized flow model.

For traditional high speed applications, accurate solutions for non-equilibrium gas flows can be obtained by the direct solution of the Boltzmann equation or by using the direct simulation Monte Carlo (DSMC) method. However, for low speed gas flows, these methods are impracticable with current computer technology. The DSMC method suffers from large statistical scatter, while the direct solution of the Boltzmann equation is very complex $[1,2]$. Meanwhile, extended hydrodynamic models, including Grad's 13-moment method and the Burnett equations, have failed to produce satisfactory results for gas flows in the transition regime [3]. Although significant progress has been made in coupling the Navier-Stokes equations with the Bhatnagar-Gross-Krook (BGK) model [4], developing the information preservation method for DSMC [5,6], and reducing the statistical scatter associated with 
Monte Carlo methods [7, 8], there is still no comprehensive and numerically-economical model for simulating low speed rarefied gas flows at the micro-scale and nano-scale.

In the last two decades, the lattice Boltzmann (LB) method has been successfully developed as an alternative to Navier-Stokes solvers. Recently, the LB methodology has been used to model non-equilibrium gas flow in regimes where the Navier-Stokes equations are no longer valid [9-27]. One important advantage of the LB methodology is that it is kinetic in nature, so the method does not suffer from closure and boundary condition problems associated with high-order continuum methods, such as Grad's method of moments [28]. In this paper, we will discuss the progress made in modeling non-equilibrium gas flow using the LB approach, with emphasis on our high-order LB model. Furthermore, we will test these models to see whether they can capture the nonlinear constitutive relation for the stress in the Knudsen layer, which is a critical feature for non-equilibrium flow.

\section{STANDARD LATTICE BGK MODEL}

With appropriate boundary conditions, LB models have demonstrated they can capture velocity slip and temperature jump at a solid surface [25]. As Sbragaglia et al. [29] have observed, the LB model has the potential to go beyond the Navier-Stokes equations. In this paper, we show that the standard lattice BGK model can describe not only the velocity slip but also some high-order rarefaction phenomena which the Navier-Stokes equations fail to predict. The details of the model are widely available in literature e.g. [30].

$$
\frac{\partial f_{k}}{\partial t}+e_{k i} \frac{\partial f_{k}}{\partial x_{i}}=-\frac{f_{k}-f_{k}^{e q}}{\phi}+\frac{\left(e_{k i}-u_{i}\right) F_{i}}{c_{s}^{2} \rho} f_{k}^{e q}
$$

where $f_{k}$ is the velocity distribution function, $f_{k}^{e q}$ is the distribution function at equilibrium, $e_{k i}$ is the lattice velocity, $u_{i}$ is the macroscopic velocity, $c_{s}$ is the sound speed of the lattice fluid, $\rho$ is the density, $\phi$ is the relaxation time, and $F_{i}$ is an applied force.

After discretizing Eq. (2), we obtain

$$
f_{k}\left(\mathbf{x}+\mathbf{e}_{k} \delta t, t+\delta t\right)-f_{k}(\mathbf{x}, t)=-\frac{1}{\tau}\left[f_{k}(\mathbf{x}, t)-f_{k}^{e q}(\mathbf{x}, t)\right]+\delta t \frac{\left(e_{k i}-u_{i}\right) F_{i}}{c_{s}^{2} \rho} f_{k}^{e q}(\mathbf{x}, t)
$$

where $\tau=\phi / \delta t$ is the nondimensional relaxation time and $\delta t$ is the time step.

For a two dimensional, nine-velocity lattice model (D2Q9), the equilibrium distribution 


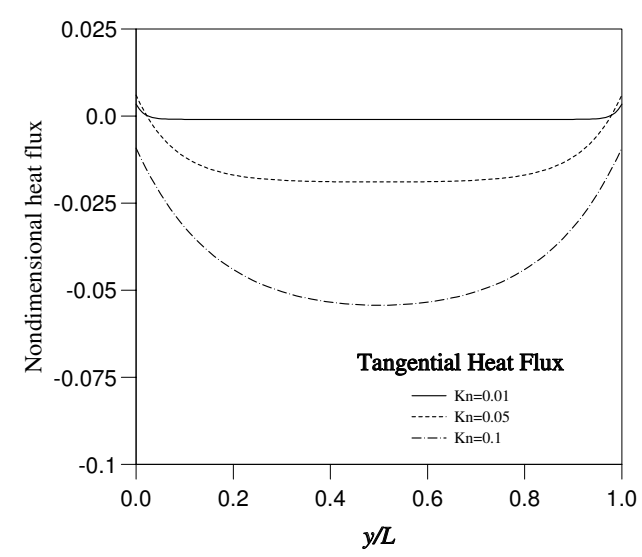

FIG. 1: Tangential heat flux in Poiseuille flow with a uniform temperature field.

function can be expressed as:

$$
\begin{gathered}
f_{k}^{e q}=\rho \omega_{k}\left[1+\frac{e_{k i} u_{i}}{c_{s}^{2}}+\frac{\left(e_{k i} u_{i}\right)^{2}}{2 c_{s}^{4}}-\frac{u_{i} u_{i}}{2 c_{s}^{2}}\right], \\
\omega_{0}=\frac{4}{9} ; \quad \omega_{k}=\frac{1}{9}, k=1,2,3,4 ; \quad \omega_{k}=\frac{1}{36}, k=5,6,7,8,
\end{gathered}
$$

The lattice velocities, $e_{k}$, are given by

$$
\begin{array}{ll}
e_{0}=0, \\
e_{k}=\left[\cos \left(\frac{(k-1) \pi}{2}\right), \sin \left(\frac{(k-1) \pi}{2}\right)\right] c, & k=1-4, \\
e_{k}=\left[\cos \left(\frac{(k-5) \pi}{2}+\frac{\pi}{4}\right), \sin \left(\frac{(k-5) \pi}{2}+\frac{\pi}{4}\right)\right] \sqrt{2} c, & k=5-8,
\end{array}
$$

where $c_{s}^{2}=c^{2} / 3, c=\sqrt{3 R T}$, and $R$ is the gas constant. The heat flux $Q$ can be calculated by the non-equilibrium part of the distribution function as

$$
Q_{i}=\left(1-\frac{1}{2 \tau_{t}}\right) \sum_{k} \frac{\left(e_{k i}-u_{i}\right)^{2}}{2}\left[f_{k}(\mathbf{x}, t)-f_{k}^{e q}(\mathbf{x}, t)\right] e_{k i},
$$

where $\tau_{t}$ can be related to $\tau$ via $\left(\tau_{t}-0.5\right) \operatorname{Pr}=\tau-0.5$ and $\operatorname{Pr}$ is the Prandtl number. The kinetic boundary conditions given by Ansumali and Karlin [31] are used in the simulations.

For a pressure driven flow in a channel, the Navier-Stokes equations will predict a zero tangential heat flux if there is no temperature variation in the channel. However, by directly solving the Boltzmann equation, Ohwada et al. [32] have observed a tangential heat flux, which can also be captured by the standard lattice BGK model. In a Poiseuille flow with the two plates at the same temperature, we examine the role of the Knudsen number in the 
tangential heat flux. In Fig. 1, the tangential heat flux $Q_{t}$ is nondimensionalized by $\rho u_{m} c^{2}$, where $u_{m}$ is the mean channel velocity. When the Knudsen number is small $(K n=0.01$ and 0.05), the tangential heat flux at the wall is positive and decreases away from the wall. At $K n=0.05$, the heat flux at the wall has increased but then decreases and goes negative a short distance from the wall. If the Knudsen number increases to 0.1, the tangential heat flux in the whole flow field is negative, including at the wall, and the magnitude has become become substabtially larger. This phenomenon has also been observed by Ohwada et al. [32] using the linearized Boltzmann equation. For low speed flow with a uniform temperature field, the total tangential heat flux, $Q_{t}$, is caused by the work done by the the viscous shear $Q_{s}$ and the pressure gradient, $Q_{p}$. According to Grad's 13 moment model [33], $Q_{s}$ is proportional to the gradient of the shear stress, which is positive for this case, and $Q_{p}$ is proportional to the pressure gradient, which is negative. When the Knudsen number is small, $K n=0.01$, the small amount of slip at the wall leads to a large wall shear stress and a large gradient of shear stress, so that the magnitude of $Q_{s}$ is relatively important. When the Knudsen number increases, the larger slip velocity leads to a smaller shear stress and also its gradient. Consequently, $Q_{p}$ becomes increasingly dominant which leads to the total tangential heat flux $Q_{t}$ going negative at a Knudsen number of 0.1. This highorder rarefaction phenomenon cannot be captured by the Navier-Stokes equations, which indicates that LB methods can go beyond the Navier-Stokes level. However, the above lattice BGK model fails to properly predict the flow characteristics in the Knudsen layer which is illustrated in Fig. 2. As high-order moments are important in describing flow in the Knudsen layer, new high-order LB models are required for highly non-equilibrium gas flows.

\section{HIGH-ORDER LB MODELS}

To accurately describe rarefied gas dynamics beyond the Navier-Stokes level, high-order LB models have been proposed [21, 37]. One reason the standard lattice BGK model fails to capture the non-linear constitutive behavior is that it only retains velocity terms up to 2 nd-order in the Hermite expansion of the equilibrium distribution function. This is not sufficient to accurately describe stresses in isothermal flows [21]. To capture non-equilibrium effects, we should retain up to 4th-order terms in the Hermite expansion [21]. Here, we can 


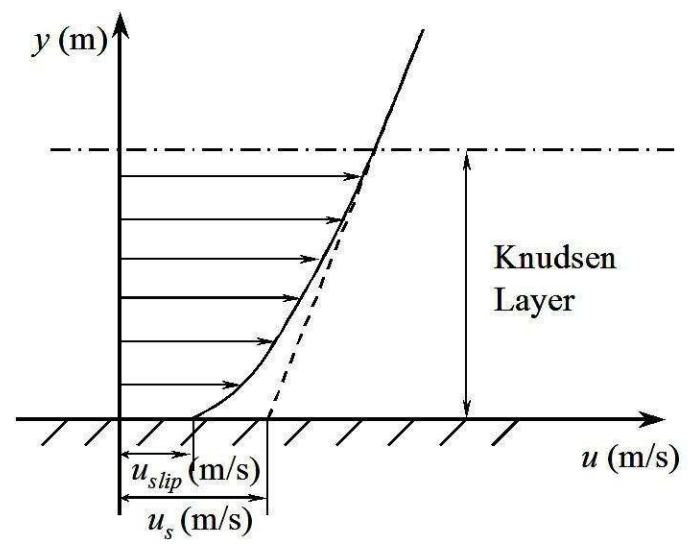

FIG. 2: Schematic diagram showing the microscopic slip $\left(u_{s l i p}\right)$ and macroscopic slip $\left(u_{s}\right)$ for Kramers' problem [34]. Actual velocity profile (-) and velocity profile predicted by the standard lattice BGK model (--) within the Knudsen layer.

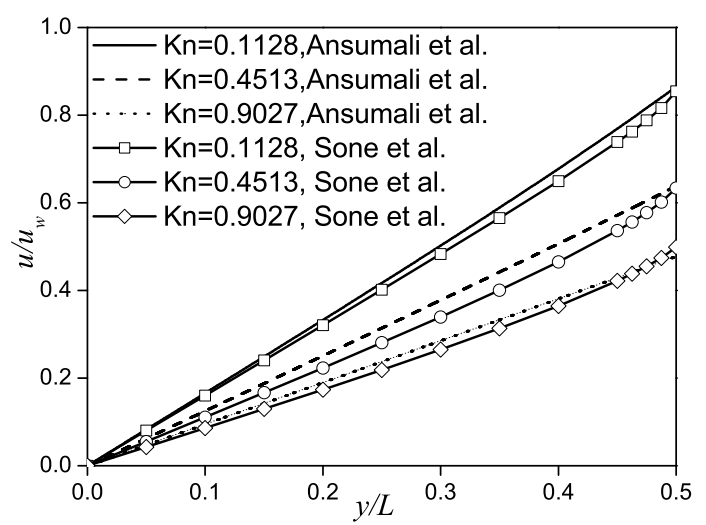

(a)

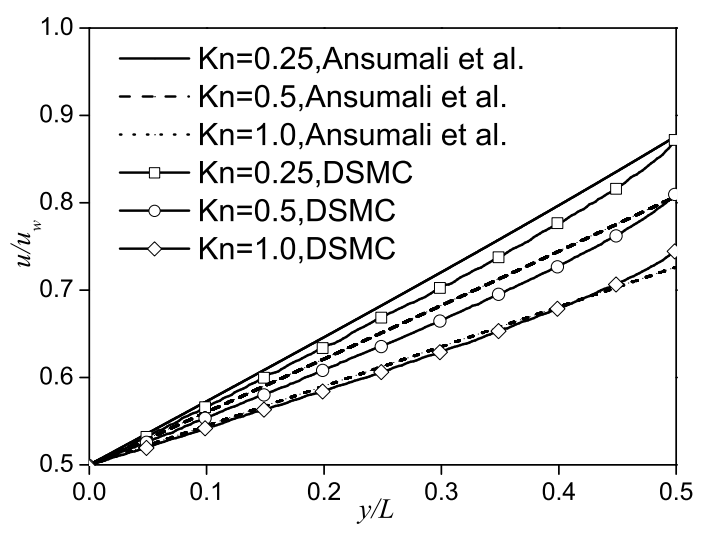

(b)

FIG. 3: Nondimensional velocity profiles for planar Couette flow, where $u_{w}$ is the velocity of the top plate. Comparison of the analytical solution of Ansumali et al. [35] with a) the linearized Boltzmann solution obtained by Sone et al. [36]; b) DSMC data.

examine whether such a model can capture the flow characteristics in the Knudsen layer by applying it to a simple isothermal Couette flow. For the D2Q16 model, Ansumali et al. [35] have derived an analytical solution for the velocity profile in planar Couette flow. In Fig. 3, their analytical solution is compared with our DSMC results and the solution of the linearized Boltzmann equation given by Sone et al. [36]. For the Couette flows presented in 


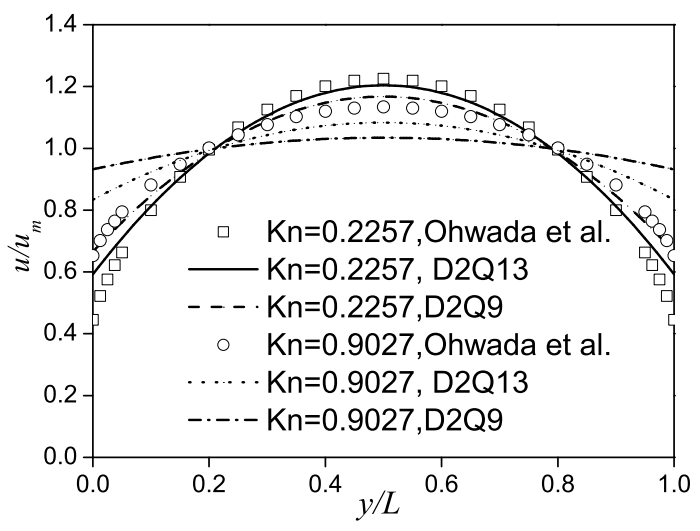

FIG. 4: Nondimensional velocity profiles for Poiseuille flow at Knudsen numbers of 0.2257 and 0.9027, where $u_{m}$ is the average velocity. The LB models are compared with the solution of the linearized Boltzmann equation obtained by Ohwada et al. [32].

this paper, the bottom plate is located at $y=-L / 2$ and moves with a velocity $-u_{w}$ and the top plate, at $y=L / 2$, moves with a velocity $u_{w}$. Only the upper half of the flow domain is shown in Fig. 3. It is clear that the D2Q16 LB model is not able to resolve the Knudsen layer in a quantitatively accurate way.

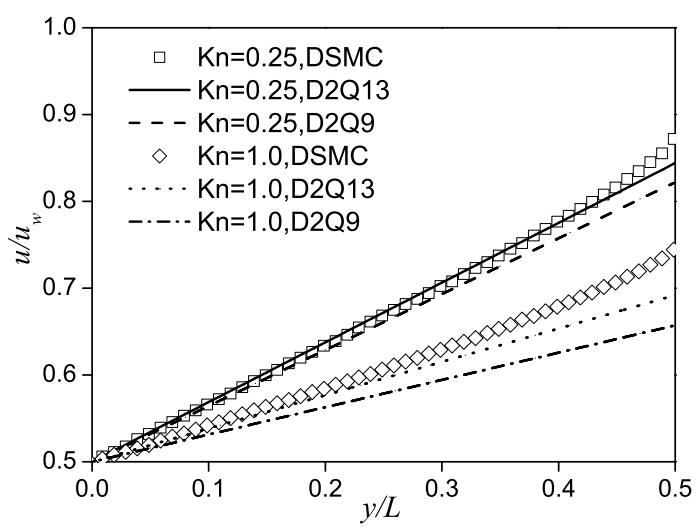

FIG. 5: Nondimensional velocity profiles for planar Couette flow, where $u_{w}$ is the velocity of the moving top plate. The LB models are compared with DSMC data.

Although Ansumali et al. [35] have demonstrated that the high-order LB models have improved current capability, the nonlinear consitutive relation between stress and strain rate 
in the Knudsen layer is still not captured. Furthermore, the roots of the 4th-order Hermite polynomials are irrational, so that the discrete velocities cannot match lattice nodes. Additional effort, such as point-wise interpolation [38], is therefore required and this dramatically increases the computational cost (because it essentially becomes an off-lattice discrete velocity method for solving the kinetic Boltzmann equation). Worse still, these high-order LB models with large numbers of discrete velocities are not numerically stable [39]. To ensure that the computational particles can move on the lattice nodes in any time step, a rational-number approximation technique, a novel concept recently demonstrated in 1D by Chikatamarla and Karlin [37], is used to construct a simpler lattice structure to avoid pointwise interpolation. In a similar spirit, we have developed a simplified high-order D2Q13 model which is described below:

$$
\begin{array}{r}
f_{k}^{e q}=\rho \omega_{k}\left[1+\frac{e_{k i} u_{i}}{c_{s}^{2}}+\frac{\left(e_{k i} u_{i}\right)^{2}}{2 c_{s}^{4}}-\frac{u_{i} u_{i}}{2 c_{s}^{2}}+\frac{\left(e_{k i} u_{i}\right)^{3}}{2 c_{s}^{6}}-\frac{3\left(e_{k i} u_{i}\right)\left(u_{i} u_{i}\right)}{2 c_{s}^{4}}\right], \\
\omega_{0}=\frac{3}{8} ; \quad \omega_{k}=\frac{1}{12}, k=1-4 ; \quad \omega_{k}=\frac{1}{16}, k=5-8 ; \quad \omega_{k}=\frac{1}{96}, k=9-12,
\end{array}
$$

where $c_{s}^{2}=c^{2} / 2$ and $c=\sqrt{2 R T}$. The lattice velocities, $e_{k}$, are given by

$$
\begin{array}{ll}
e_{0}=0 \\
e_{k}=\left[\cos \left(\frac{(k-1) \pi}{2}\right), \sin \left(\frac{(k-1) \pi}{2}\right)\right] c, & k=1-4, \\
e_{k}=\left[\cos \left(\frac{(k-5) \pi}{2}+\frac{\pi}{4}\right), \sin \left(\frac{(k-5) \pi}{2}+\frac{\pi}{4}\right)\right] \sqrt{2} c, & k=5-8, \\
e_{k}=\left[\cos \left(\frac{(k-1) \pi}{2}\right), \sin \left(\frac{(k-1) \pi}{2}\right)\right] 2 c, & k=9-12 .
\end{array}
$$

Figure 4 clearly shows that our D2Q13 performs much better than the D2Q9 model for Poiseuille flow when compared with the solution of the linearized Boltzman equation. The improved performance of our D2Q13 model is also demonstrated in Fig. 5, even though it fails to capture the flow characteristics in the Knudsen layer.

\section{MODIFICATIONS}

The currently available high-order models are not sufficiently accurate to describe flow behavior in the Knudsen layer. Other work to improve LB model predictions has appeared in the literature. Examples include regularization methods that add an additional term 


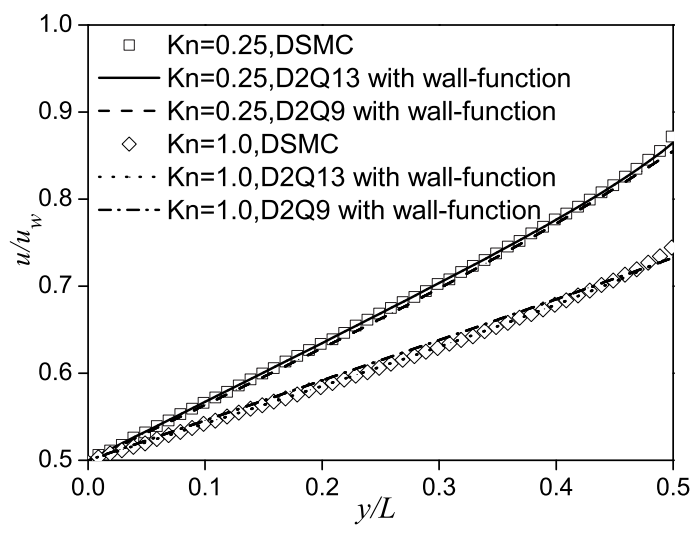

FIG. 6: Nondimensional velocity profiles for planar Couette flow, where $u_{w}$ is the velocity of the top plate. The LB model with the wall-function is compared with DSMC data.

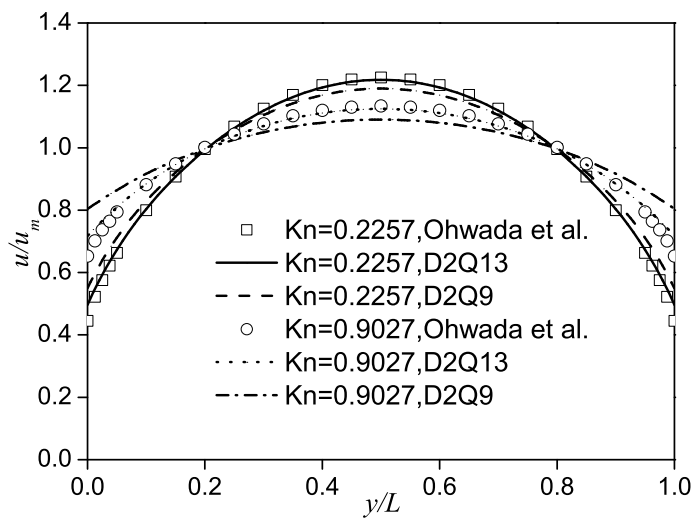

FIG. 7: Nondimensional velocity profiles for Poiseuille flows at Knudsen numbers of 0.2257 and 0.9027, where $u_{m}$ is the average velocity. The LB model with the wall-function is compared to the solution of the linearized Boltzmann equation obtained by Ohwada et al. [32]

considering the omitted high-order moment effects $[23,26]$, which is in the same spirit as the regularized moment models [40, 41]. Szalmás [27] recently proposed a modification to implement the effect of high-order moments through a fast relaxation mode, which is similar to the split of fast and slow moments proposed by Gorban and Karlin [42]. Although the above modifications have been shown to improve the capability of lattice Boltzmann models, they are still at an early stage and further work is clearly required. We have recently proposed a wall-function approach for the standard lattice BGK model which can accurately describe 
the nonlinear velocity profiles in the Knudsen layer for simple planar Couette flows [22, 25]. The collisions between gas molecules and the wall means that the mean free path of a gas will be reduced. As a consequence, the bulk value does not accurately reflect the mean free path in the Knudsen layer. The gas mean free path in the near-wall region can be approximated as [22]

$$
\lambda_{e}=\frac{\lambda}{1+0.7 e^{-C y / \lambda}},
$$

where $\lambda_{e}$ is the effective mean free path and $C$ is a constant. With increasing Knudsen number, the Knudsen layers will start to overlap. For the flow between two parallel plates considered here, the effective mean free path in the overlapped Knudsen layers is assumed to be

$$
\lambda_{e}=\frac{\lambda}{1+0.7\left(e^{-C y / \lambda}+e^{-C(L-y) / \lambda}\right)},
$$

where $y$ is the distance from the lower wall and $L-y$ is the distance from the upper wall. With our high-order model given in Eqs. $(7,8)$, the wall-function approach can enable the D2Q13 model to quantitatively simulate not only planar Couette flow but also pressuredriven flows. Figure 6 shows that the nonlinear velocity profiles predicted by our model for Knudsen numbers of 0.25 and 1.0 are in excellent agreement with the solution of the linearized Boltzmann equation obtained by Ohwada et al. [32]. In addition, Fig. 7 illustrates how our D2Q13 model, with the effect of the wall taken into consideration, can produce accurate results that are close to the direct solution of the Boltzmann equation at a fraction of the computational cost.

\section{SUMMARY}

The standard lattice BGK method is able to predict some high-order rarefaction phenomena. However, high-order lattice Boltzmann models, based on the BGK equation, cannot capture the flow characteristics in the Knudsen layer with sufficient accuracy. Although some modifications of the lattice BGK model appear to improve the model's performance, significant work is still required for future high-order LB methods to be quantatitively accurate. Meanwhile, with a simple wall-function to consider the wall effect on the gas mean free path, our computationally efficient high-order D2Q13 LB model is able to describe nonequilibrium flows with accuracy comparable to both DSMC methods and the direct solution of the linearized Boltzmann equation. 


\section{ACKNOWLEDGMENTS}

The authors are grateful to helpful discussions with Drs Xiao-Jun Gu and Robert Barber at Daresbury Laboratory, and Professor Jason Reese at Strathclyde University. This work is financially supported by UK Engineering and Physical Sciences Research Council (EPSRC) under grant no. EP/D07455X/1. Additional support was provided by EPSRC under the auspices of Collaborative Computational Project 12.

[1] N. G. Hadjiconstantinou, A. Garcia, M. Bazant, and G. He, J. Comput. Phys. 187, 274 (2003).

[2] F. Sharipov, L. M. G. Cumin, and D. Kalempa, Euro. J. Mech. B-Fluid 23, 899 (2004).

[3] C. L. Bailey, R. W. Barber, and D. R. Emerson, in European Congress on Computational Methods in Applied Sciences and Engineering, ECCOMAS 2004, edited by P. Neittaanmäki, T. Rossi, S. Korotov, E. Oñate, J. Périaux, and D. Knörzer (Jyväskylä, Finland, 2004).

[4] K. Xu and Z. Li, J. Fluid Mech. 513, 87 (2004).

[5] J. Fan and C. Shen, J. Comp. Phys. 167, 393 (2001).

[6] Q. Sun and I. D. Boyd, pp. AIAA-2005-4828 (2005).

[7] L. L. Baker and N. G. Hadjiconstantinou, Phys. Fluids 17, 051703 (2005).

[8] J. Chun and D. L. Koch, Phys. Fluids 17, 107107 (2005).

[9] X. B. Nie, G. Doolen, and S. Chen, J. Stat. Phys. 107, 279 (2002).

[10] C. Y. Lim, C. Shu, X. D. Niu, and Y. T. Chew, Phys. Fluids 14, 2299 (2002).

[11] X. D. Niu, C. Shu, and Y. T. Chew, Europhys. Lett. 67, 600 (2004).

[12] T. Lee and C.-L. Lin, Phys. Rev. E 71, 046706 (2005).

[13] Y. H. Zhang, R. S. Qin, and D. R. Emerson, Phys. Rev. E 71, 047702 (2005).

[14] Y. H. Zhang, R. S. Qin, Y. H. Sun, R. W. Barber, and D. R. Emerson, J. Stat. Phys. 121, 257 (2005).

[15] G. H. Tang, W. Q. Tao, and Y. L. He, Phys. Rev. E 72, 056301 (2005).

[16] G. H. Tang, W. Q. Tao, and Y. L. He, Phys. Fluids 17, 058101 (2005).

[17] M. Sbragalia and S. Succi, Phys. Fluids 17, 093602 (2005).

[18] F. Toschi and S. Succi, Europhys. Lett. 69, 549 (2005). 
[19] V. Sofonea and R. F. Sekerka, J. Comp. Phys. 207, 639 (2005).

[20] V. Sofonea and R. F. Sekerka, Phys. Rev. E 71, 066709 (2005).

[21] X. Shan, X.-F. Yuan, and H. Chen, J. Fluid Mech. 550, 413 (2006).

[22] Y. H. Zhang, X. J. Gu, R. W. Barber, and D. R. Emerson, Phys. Rev. E 74, 046704 (2006).

[23] R. Zhang, X. Shan, and H. Chen, Phys. Rev. E 74, 046703 (2006).

[24] Z. L. Guo, T. S. Zhao, and Y. Shi, J. Appl. Phys. 99, 074903 (2006).

[25] Y. H. Zhang, X. J. Gu, R. W. Barber, and D. R. Emerson, EPL 77, 30003 (2007).

[26] X.-D. Niu, S.-A. Hyodo, T. Munekata, and K. Suga, Phys. Rev. E 76, 036711 (2007).

[27] L. Szalmás, EPL 80, 24003 (2007).

[28] X. J. Gu and D. R. Emerson, J. Comput. Phys. 225, 263 (2007).

[29] M. Sbragalia and S. Succi, Europhys. Lett. 73, 370 (2006).

[30] S. Chen and G. D. Doolen, Annu. Rev. Fluid Mech. 30, 329 (1998).

[31] S. Ansumali and I. V. Karlin, Phys. Rev. E 66, 026311 (2002).

[32] T. Ohwada, Y. Sone, and K. Aoki, Phys. Fluids A1, 1588 (1989).

[33] H. Struchtrup, Macroscopic transport equations for rarefied gas flows (Springer-Verlag, Berlin, 2005).

[34] H. A. Kramers, Suppl. Nuovo. Cimento 6, 297 (1949).

[35] S. Ansumali, I. V. Karlin, S. Arcidiacono, A. Abbas, and N. I. Prasianakis, Phys. Rev. Lett. 98, $124502(2007)$.

[36] Y. Sone, S. Takata, and T. Ohwada, Euro. J. Mech. B: Fluids 9, 273 (1990).

[37] S. S. Chikatamarla and I. V. Karlin, Phys. Rev. Lett. 97, 190601 (2006).

[38] X. He, L. S. Luo, and M. Dembo, J. Comp. Phys. 129, 357 (1996).

[39] S. Succi, I. V. Karlin, and H. Chen, Rev. Modern Phys. 74, 1203 (2002).

[40] I. V. Karlin, A. N. Gorban, G. Dukek, and T. F. Nonnenmacher, Phys. Rev. E 57, 1668 (1998).

[41] H. Struchtrup and M. Torrilhon, Phys. Fluids 15, 2668 (2003).

[42] A. N. Gorban and I. V. Karlin, Physica A 360, 325 (2006). 